\title{
Author Correction: Targeted NUDT5 inhibitors block hormone signaling in breast cancer cells
}

Brent D.G. Page ${ }^{1,7 \star}$, Nicholas C.K. Valerie (1) ${ }^{1,7}$, Roni H.G. Wright ${ }^{2,3}$, Olov Wallner ${ }^{1}$, Rebecka Isaksson ${ }^{1}$, Megan Carter ${ }^{4}$, Sean G. Rudd (1) ${ }^{1}$, Olga Loseva', Ann-Sofie Jemth', Ingrid Almlöf1, Jofre Font-Mateu ${ }^{2,3}$, Sabin Llona-Minguez (10 ${ }^{1}$, Pawel Baranczewski ${ }^{5}$, Fredrik Jeppsson ${ }^{1}$, Evert Homan ${ }^{1}$, Helena Almqvist ${ }^{6}$, Hanna Axelsson ${ }^{6}$, Shruti Regmi ${ }^{6}$, Anna-Lena Gustavsson ${ }^{6}$, Thomas Lundbäck ${ }^{6}$, Martin Scobie ${ }^{1}$, Kia Strömberg ${ }^{1}$, Pål Stenmark ${ }^{4}$, Miguel Beato ${ }^{2,3} \&$ Thomas Helleday ${ }^{1 \star}$

Correction to: Nature Communications https:/doi.org/10.1038/s41467-017-02293-7, published online 17 January 2018.

The original version of this Article omitted the following from the Acknowledgements:

This work was funded by the European Research Council (695376) (T.H.).

This has now been corrected in both the PDF and HTML versions of the Article.

Published online: 01 November 2019

(i) Open Access This article is licensed under a Creative Commons Attribution 4.0 International License, which permits use, sharing, adaptation, distribution and reproduction in any medium or format, as long as you give appropriate credit to the original author(s) and the source, provide a link to the Creative Commons license,

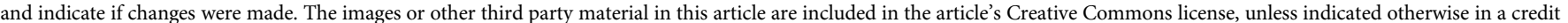

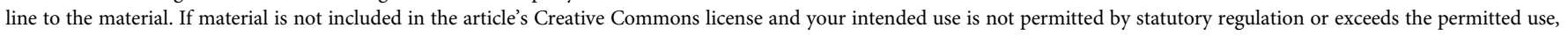
you will need to obtain permission directly from the copyright holder. To view a copy of this license, visit http://creativecommons.org/licenses/by/4.0/.

(C) The Author(s) 2019

\footnotetext{
${ }^{1}$ Science for Life Laboratory, Division of Translational Medicine and Chemical Biology, Department of Medical Biochemistry and Biophysics, Karolinska Institutet, Solna SE-171 21, Sweden. ${ }^{2}$ Centre de Regulació Genòmica (CRG), Barcelona Institute for Science and Technology, Barcelona E-09003, Spain. ${ }^{3}$ Universitat Pompeu Fabra, Barcelona E-08003, Spain. ${ }^{4}$ Department of Biochemistry and Biophysics, Stockholm University, Stockholm SE-106 91, Sweden. ${ }^{5}$ Uppsala University Drug Optimization and Pharmaceutical Profiling Platform, Department of Pharmacy, Uppsala University, Uppsala SE-751 23, Sweden. ${ }^{6}$ Chemical Biology Consortium Sweden, Science for Life Laboratory, Division of Translational Medicine and Chemical Biology, Department of Medical Biochemistry and Biophysics, Karolinska Institutet, Solna SE-171 21, Sweden. ${ }^{7}$ These authors contributed equally: Brent D.G. Page, Nicholas C.K. Valerie. *email: brent.page@scilifelab.se; thomas.helleday@scilifelab.se
} 